\title{
Long term therapy with Testosterone Undecanoate in Type 2 Diabetes was associated with reduced all-cause mortality but no significant improvement in conventional risk factors for CVD.
}

G Hackett, S Ramachandran, R Strange, P Jones.

\section{BACKGROUND}

Low testosterone levels are found in around $40 \%$ of men with type 2 diabetes (T2DM) and are associated with visceral adiposity, increased insulin resistance and features of the Metabolic syndrome (MetS). Testosterone Replacement Therapy, in short term RCTs, has been shown to improve insulin resistance, increase lean muscle mass and improve components of MetS.

PURPOSE

To assess the impact of long term testosterone replacement with long acting Testosterone Undecanoate (TU) on metabolic parameters in a 4 years longitudinal study involving a cohort of 857 men with T2DM from the BLAST Study.

\section{METHODS}

The T2DM male populations of 5 general practices were screened between April 2007 and April 2009, recruiting 2000 men with baseline Testosterone of $12 \mathrm{nmol} / \mathrm{l}$ or less or Free Testosterone 0.24 $\mathrm{nmol} / \mathrm{l}$ or less for a 30 week double blind placebo controlled randomised study of TU, with 52 weeks open label follow on with free of charge medication. Long term follow-up data after a mean of 4 years, with subsequent NHS prescriptions, was collected from the general practice records. Patients were divided into 4 groups; A. Normal $T$ (untreated) $n=320$. B Low $T$ (untreated) $n=365$. C. Low $T$ (treated continuously) $n=78$ and $D$. Low $T$ (treatment discontinued) $n=97$. The primary end point was diabetes control as assessed by HbA1c. Secondary endpoints were weight change, BMI, lipids and adverse events.

\section{RESULTS}

Across all 4 groups, there were no significant change in $\mathrm{HbA1c}$, weight, $\mathrm{BMI}$, and lipids as assessed by paired $t$ test adjusted for age. There were minor reductions in blood pressure seen in all 4 groups. Men treated continuously were younger ( 54 v 65), and more likely to be taking a PDE5 inhibitor (55\% $v 15 \%)$, suggesting that sexual function was a major driver for ongoing prescriptions. All-cause mortality rates over 4 years were as follows A 36/320 (11.25\%, B 61/362 (16.85\%). C 0/75 (0\%). D $6 / 97$ (6.19\%). Cox regression analysis between groups was not possible as no patients died in the continuous therapy group.

\section{CONCLUSIONS}

Over 4 years of follow up of 857 men with T2DM, TU therapy was not associated with improved $\mathrm{HbA1c}$, weight, BMI, and lipids. There was significantly increased all mortality in men with low $T$ that was untreated compared with men with normal $T$ at baseline. TU therapy was associated with a reduction in all-cause mortality and none of 75 men on continuous therapy died in the 4 years period. These findings confirm earlier studies suggesting that long term therapy is safe and suggests 
that reductions in all-cause mortality might be due to factors other than reduction of conventional markers of CVD risk. 